superior increase in statins prescription was observed ( $36 \%$ vs. $13 \%, p=0.000)$, as well in hypertension and aspirin treatment. However, no differences were noticed in the changes in disease-related data that occur during the time the study was carried out.

Conclusion: This real-world study demonstrates that incorporating carotid ultrasound into routine clinical practice in RA patients is effective in achieving LDL-cholesterol targets for the prevention of cardiovascular disease. This effect is not only mediated by the increase in the prescription of statins, but also by the better control of other cardiovascular risk factors that occurred after carotid ultrasound evaluation.

Disclosure of Interests: None declared

DOI: 10.1136/annrheumdis-2021-eular.1883

\section{POS0534 $\quad$ THE PREVALENCE OF HYPERTENSION AND ITS POTENTIAL ASSOCIATED FACTORS IN CHINESE PATIENTS WITH RHEUMATOID ARTHRITIS}

Y. W. Zou ${ }^{1}$, C. Chen ${ }^{2}$, J. Lin ${ }^{1}$, J. D. Ma ${ }^{2}$, Y. Y. Zou ${ }^{1}$, L. Dai ${ }^{1}{ }^{1}$ Sun Yat-Sen Memorial Hospital, Sun Yat-Sen University, Department of Rheumatology, Guangzhou, China; ${ }^{2}$ Sun Yat-Sen Memorial Hospital, Sun Yat-Sen University, Department of Rheumatology, Guangzhou, China

Background: Cardiovascular disease (CVD) is the leading cause of mortality in patients with rheumatoid arthritis (RA), and hypertension is a modifiable risk-factor for CVD. In addition, comorbidities may shorten the life span of RA patients, which appears to be the consequence of an increased prevalence of CVD. RA patients with comorbidities might not be given the equal treatment in daily practice compared with patients without comorbidities.

Objectives: To investigate the prevalence of hypertension and its associated factors in Chinese RA patients.

Methods: Consecutive patients with RA were recruited from August 2015 to September 2019 at Department of Rheumatology, Sun Yat-sen Memorial Hospital. Demographic and clinical characteristics were collected including indicators of disease activity, functional assessment and radiographic assessment and CV-related comorbidities.

Results: There were 674 RA patients recruited with $82.3 \%$ female and mean age $49.9 \pm 13.0$ years. The prevalence rate of hypertension in RA patients was $32.9 \%$, followed by dyslipidemia $(9.9 \%)$, type 2 diabetes $(8.8 \%)$, hyperuricemia $(8.5 \%)$, fatty liver disease $(8.0 \%)$, CVD $(6.2 \%)$ and chronic kidney disease $(3.3 \%)$. The prevalence of hypertension was $38.7 \%$ and $31.7 \%$ in male and female RA patients respectively $(P=0.144)$. Further age stratification showed no hypertension in RA patients at $16-19$ years, $2.0 \%$ at $20-29$ years and $8.1 \%$ at $30-39$ years. The prevalence of hypertension greatly increased after 40 years old with $24.5 \%, 38.6 \%, 54.9 \%$, and $63.6 \%$ in RA patients of $40-49,50-59,60-69, \geq 70$ years old respectively, and there was no significant difference between male and female RA patients of different age (Figure 1). Compared with those without hypertension, RA patients with hypertension had advanced age (57.3 \pm 9.3 years vs. $46.3 \pm 13.0$ years), long-standing disease duration (median 60 months vs. 48 months), higher disease activity indicators [including PtGA (median 4 vs. 3), PrGA (median 3 vs. 3) and ESR (median $33 \mathrm{~mm} / \mathrm{h}$ vs. $27 \mathrm{~mm} / \mathrm{h}$ )], higher functional indicator [HAQ-DI (median 0.25 vs. 0.13 )], worse joint destruction [JE subscore (median 10 vs. $6)$ ] and higher proportions of comorbidities [including type 2 diabetes (14.9\% vs. $5.8 \%)$, hyperuricemia $(13.1 \%$ vs. $6.1 \%)$, chronic kidney disease $(6.3 \%$ vs. $1.8 \%$ ), dyslipidemia (15.8\% vs. $7.1 \%)$, CVD (10.4\% vs. $4.2 \%$ ) and fatty liver disease $(13.5 \%$ vs. $5.3 \%)$, all $P<0.005$ ]. Multivariate logistic regression analysis showed that comorbidities including hyperuricemia $(O R=1.977,95 \% \mathrm{Cl}$ : 1.002-3.900), dyslipidemia $(O R=1.903,95 \% \mathrm{Cl}: 1.102-3.288)$ and fatty liver disease $(O R=2.335,95 \% \mathrm{Cl}: 1.278-4.265)$ were associated factors of hypertension after adjustment for age and gender
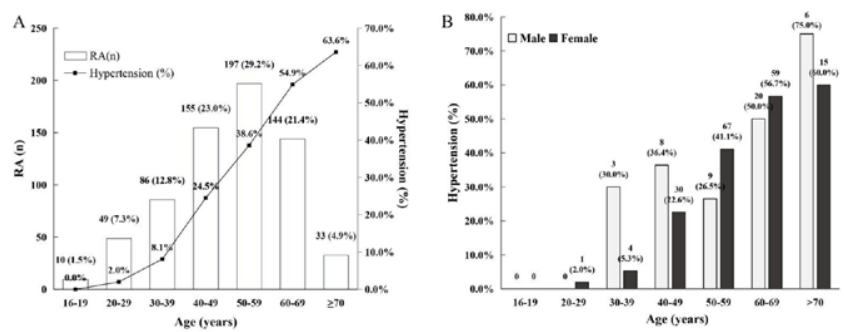

Figure 1. The prevalence of hypertension in different age and sex groups of RA patients.

Conclusion: Hypertension is the most common CV-related comorbidity in Chinese RA patients which is associated with hyperuricemia, dyslipidemia and fatty liver disease. Detection and management of hypertension and other CV-related comorbidities in RA patients should be emphasized.

\section{Fund program:}

National Natural Science Foundation of China (81801605, 81801606, 81971527); Guangdong Natural Science Foundation (2018A030313541 2018A030313690, 2019A1515011928); Guangzhou Science and Technology Program (201904010088); Guangdong Basic and Applied Basic Research Foundation (2020A1515110061); Guangdong Medical Scientific Research Foundation (A2018062)

Disclosure of Interests: None declared

DOI: 10.1136/annrheumdis-2021-eular.2078

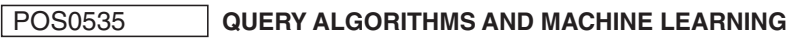 METHODS AS TOOLS TO IDENTIFY COMORBIDITIES IN LARGE-SCALE FREE-TEXT BASED FIELDS: A CASE-REPORT}

D. Rohrich ${ }^{1}$, T. Maarseveen ${ }^{2}$, A. de Boer ${ }^{1}$, C. van den Ende ${ }^{1}$, A. den Broeder ${ }^{1}$, C. Popa ${ }^{1}$, R. Knevel ${ }^{2}{ }^{1}$ Sint Maartenskliniek, Rheumatology, Ubbergen, Netherlands; ${ }^{2}$ Leiden University Medical Center (LUMC), Rheumatology, Leiden, Netherlands

Background: Inflammatory rheumatic conditions (IRC) are associated with comorbidity, two most important being cardiovascular diseases (CVD) and infections [1, 2]. A crucial initial step when proceeding with studying CVD and infections in these patients is identification of events. The large scale EHR datasets enable studies to assess low incident and clinically important events, but requires both accurate as well as efficient data extraction. Studying Electronic Health Records (EHR) using query-based algorithms (QBA) and machine learning algorithms (MLA) offers a valuable tool to screen large-scale collections for rare events, thus replacing resource intensive manual chart review.

Objectives: To explore the (comparative) usefulness of QBA and MLA to identify CVD and infection events in EHR free-text data in patients with chronic IRC.

Methods: To independently develop and validate the algorithms we used two EHR databases, i.e. a training set with psoriatic arthritis patients $(\mathrm{N}=977$, dataset $A$, Golden Standard) and a validation set of rheumatoid arthritis patients $(\mathrm{N}=1098$, dataset $\mathrm{B})$. Using both QBA and MLA, we aimed to identify (yes/no and timing) CVD and infections. We assessed the performances of the algorithms by calculating the specificity, sensitivity, positive predictive value (PPV) and the negative predictive value (NPV), respectively.

Results: In the final performance analysis on dataset $B$, both QBA and MLA showed a high performance in identifying CVD (sensitivity, specificity, PPV, NPV for QBA $((95 \% \mathrm{Cl})=0.69(0.66-0.72), 0.99(0.96-1.02), 0.84(0.81-0.87)$ $0.98(0.95-1.01))$ and for MLA (sensitivity $=0.69(0.66-0.72), 0.98$ (0.95-1.01), 0.68 (0.65-0.71), 0.98 (0.95-1.01), respectively) Infections showed similar performance (QBA sensitivity, specificity, PPV, NPV is $0.64(0.61-0.67), 0.96(0.93$ $0.99), 0.66$ (0.63-0.69), 0.96 (0.93-.0.99) and for MLA = 0.61 (0.58-0.64), 0.93 (0.90-0.96), 0.49 (0.46-0.52), 0.96 (0.93-0.99), respectively). For infections the specificity was slightly higher for QBA relative to MLA.

Conclusion: We found a consistent high performance of both the QBA and MLA algorithms for the identification of CVD and infections in our free text EHR of patients with chronic IRC (Table 1). The performance of QBA highly depends on the domain knowledge of the builders, which might allow it to outperform a Gold Standard. MLA is efficient as it does not require any domain knowledge, but its performance is restricted by the quality of the Gold Standard.

Table 1. Overlap and differences in event identification of the query based and machine learning based algorithms compared to the Gold Standard dataset B (chart review by specialized health care professional). The results of non-event and event identification by the algorithms is represented in numbers of events and percentages of the total non-event or event.

Dataset B Cardiovascular events

\begin{tabular}{|c|c|c|c|c|}
\hline & QBA - & ML - & QBA + & ML + \\
\hline $\mathrm{GS}-(\mathrm{N}=2123)$ & $2102(99.0 \%)$ & 2071 (97.6\%) & $21(0.99 \%)$ & $52(2.45 \%)$ \\
\hline $\mathrm{GS}+(\mathrm{N}=159)$ & $49(30.1 \%)$ & $49(30.1 \%)$ & $110(69.2 \%)$ & $110(69.2 \%)$ \\
\hline \multicolumn{5}{|c|}{ Total events GS =2282 } \\
\hline & QBA - & ML - & QBA + & $M L+$ \\
\hline$G S-(N=2053)$ & 1979 (96.4\%) & $1910(93.0 \%)$ & $74(3.6 \%)$ & $143(7.0 \%)$ \\
\hline $\mathrm{GS}+(\mathrm{N}=228)$ & $83(36.4 \%)$ & $89(39.0 \%)$ & 145 (63.6\%) & $139(61.0 \%)$ \\
\hline Total events GS & & & & \\
\hline
\end{tabular}

(QBA = Query Based Algorithm, MLA = Machine Learning Algorithm, GS - = Non-event in Gold Standard dataset B, GS + = Event in Gold Standard dataset $B$ ) 